\title{
Intermediate Templates Guided Groupwise Registration of Diffusion Tensor Images
}

\author{
Hongjun Jia ${ }^{1}$, Pew-Thian Yap ${ }^{1}$, Guorong Wu ${ }^{1}$, Qian Wang ${ }^{1,2}$, and Dinggang Shen ${ }^{1, *}$ \\ Department of Radiology and BRIC, University of North Carolina at Chapel Hill, NC 27599, U.S.A. \\ Department of Computer Science, University of North Carolina at Chapel Hill, NC 27599, U.S.A.
}

\section{Abstract}

Registration of a population of diffusion tensor images (DTIs) is one of the key steps in medical image analysis, and it plays an important role in the statistical analysis of white matter related neurological diseases. However, pairwise registration with respect to a pre-selected template may not give precise results if the selected template deviates significantly from the distribution of images. To cater for more accurate and consistent registration, a novel framework is proposed for groupwise registration with the guidance from one or more intermediate templates determined from the population of images. Specifically, we first use a Euclidean distance, defined as a combinative measure based on the FA map and ADC map, for gauging the similarity of each pair of DTIs. A fully connected graph is then built with each node denoting an image and each edge denoting the distance between a pair of images. The root template image is determined automatically as the image with the overall shortest path length to all other images on the minimum spanning tree (MST) of the graph. Finally, a sequence of registration steps is applied to progressively warping each image towards the root template image with the help of intermediate templates distributed along its path to the root node on the MST. Extensive experimental results using diffusion tensor images of real subjects indicate that registration accuracy and fiber tract alignment are significantly improved, compared with the direct registration from each image to the root template image.

\section{Keywords}

Diffusion tensor image; image registration; minimum spanning tree (MST); intermediate templates; groupwise registration; fiber tract alignment

\section{Introduction}

Diffusion tensor imaging (DTI) serves as a non-invasive in-vivo probe into brain tissues by taking advantage of water diffusion patterns which give micro-structural information especially in the white matter (Bihan et al., 2001; Pierpaoli et al., 1996). DTI has been widely applied to clinical diagnosis since white matter abnormalities can potentially be detected earlier by identifying disease-induced diffusion changes, compared to other conventional imaging techniques, such as MR T1 or T2 weighted imaging (Huang et al.,

\footnotetext{
(C) 2010 Elsevier Inc. All rights reserved.

*Corresponding author: Dinggang Shen (dgshen@med.unc.edu).
}

Publisher's Disclaimer: This is a PDF file of an unedited manuscript that has been accepted for publication. As a service to our customers we are providing this early version of the manuscript. The manuscript will undergo copyediting, typesetting, and review of the resulting proof before it is published in its final citable form. Please note that during the production process errors may be discovered which could affect the content, and all legal disclaimers that apply to the journal pertain. 
2006; Lee et al., 2008; Liu et al., 2007; Yang et al., 2008). A prerequisite for meaningful comparison of diffusion tensor images of different subjects is to bring all images into a common space by appropriate spatial alignment (Alexander, 2006). Achieving this is, however, not only technically challenging but also computationally expensive due to the intrinsic high dimensionality of DTI images and the inherent large cross-subject variance. It is also a non-trivial task to identify a common space by which the whole population under study can be well represented (Lepore et al., 2007).

One way to achieve the registration is to employ pairwise registration techniques to register each image one-by-one to a predefined template. Methods as such have been studied by many researchers (Lepore et al., 2007; Yang et al., 2008; Yap et al., 2009a, b, c, 2010; Zöllei et al., 2010). For example, Yang et al. (Yang et al., 2008) utilize the voxelwise geometry and orientation information for guiding registration. Different measurements, such as prolateness, oblateness, and sphericity, are calculated on each voxel, and the regional and rotation-invariant features for these measurements are used in a deformable correspondence matching mechanism for image alignment. More recently, two novel DTI registration algorithms, TIMER (Yap et al., 2009b, c) and its extension F-TIMER (Yap et al., 2009a, 2010), are proposed to specifically exploit features directly related to the tensor structures. In addition, two mechanisms which have been proven effective (Shen and Davatzikos, 2002) - attribute vector and driving voxels - are incorporated in TIMER and F-TIMER for more precise and robust registration.

There are, however, two potential limitations related to the pairwise registration. First, selecting a fixed template for registration can be problematic. Any existing out-ofpopulation template may not accurately characterize the population. Also, selecting an image from the population itself can be equally problematic if it differs significantly from the rest of the images in the population. Second, pairwise registration methods often cannot perform well when registering two images with significant anatomical differences. Hence, only those subjects that are close to the template could be well registered, and others (that are distant from the template) may not achieve reliable registration. In this way, the registration accuracy and robustness for the whole population could be affected.

To overcome such limitations, a number of groupwise approaches have been proposed in the literature. The goal of groupwise registration is to warp all subjects in a population towards a hidden common space simultaneously within a single framework (Barmpoutis and Vemuri, 2009; Zhang et al., 2007). Since all subjects in the data set are treated equally in the process of registration, the registration results and the final atlas are considered to be unbiased. For example, Barmpoutis and Vemuri proposed a groupwise diffusion weighted MR image registration method based on the $4^{\text {th }}$-order tensor field (Barmpoutis and Vemuri, 2009). By using a metric defined for the positive valued spherical functions, unbiased groupwise registration and atlas construction are achieved via optimizing a cost function which also takes into account the tensor reorientation during the registration. In (Zhang et al., 2007), the unbiased registration framework (Joshi et al., 2004), which was initially proposed for registering T1 images, is applied to DTI groupwise registration. Two alternating steps, atlas construction (by averaging all then-current warped images) and registration refinement (by aligning all images to the then-current atlas), are iterated until convergence. It is worth noting that in (Zhang et al., 2007) only piecewise affine registration is used to "approximate" the deformable registration. A growing body of evidence shows that groupwise methods are more consistent and accurate than direct pairwise methods. However, the inherent high dimensionality, high degrees of nonlinearity, and complexity of the optimization problem make it difficult to achieve the global optimal solution. 
When across-subject variation is large, it is generally difficult to achieve good registration by simply registering each image to a template directly. In light of this, several methods have been proposed to enhance the performance of pairwise registration methods by making use of a concept called intermediate templates (IT). Intermediate templates have been shown to be effective in the registration of T1 weighted MR brain images (Baloch and Davatzikos, 2009; Baloch et al., 2007; Hamm et al., 2009; Kim et al., 2008; Tang et al., 2009) and 2D shape correspondence (Munsell et al., 2009). To date, two different strategies have been used to implement the idea of intermediate templates, namely 1) intermediate templates construction and 2) intermediate templates selection, depending on the way that the intermediate templates are determined.

In the construction-based methods (Baloch and Davatzikos, 2009; Baloch et al., 2007; Kim et al., 2008; Tang et al., 2009), an intermediate template which does not belong to the original data set is created to aid the registration between two images. For example, in (Tang et al., 2009), intermediate templates are created by warping the template image with a set of simulated deformation fields which are learned using principal component analysis (PCA) on a set of training deformation fields. During registration, an intermediate template is automatically selected for each subject image and only the residual deformation between the subject image and the intermediate template needs to be estimated since the deformation field between the intermediate template and the final template is known. Such approaches, however, do not guarantee that the intermediate template is realistic, which may affect the registration results.

In the selection-based methods, intermediate templates are selected directly from the same dataset by building a connection pathway between each individual image and the final template. The registration is achieved by deforming each individual image with the help of intermediate templates along its respective pathway to the template. This strategy is first applied to a shape correspondence problem (Munsell et al., 2009) where a minimum spanning tree (MST), with nodes representing the shapes and edges representing the shape similarity between two connected nodes, can be used to characterize the shape distribution of the population. The root node of the built MST can be determined by selecting the node that gives a minimal overall edge length to all other nodes, or the node with the most children on the tree. In (Hamm et al., 2009), a similar approach is adopted to register a set of brain MR images. Upon learning the intrinsic manifold of the dataset, a pseudo-geodesic median image is determined from the geodesic paths computed between the individual images and the template. The utilization of intermediate templates allows us to employ a divide-and-conquer strategy where large deformations between images can be decomposed into a series of small ones, and hence reduce registration error since only nearby similar images need to be registered.

In summary, intermediate templates are helpful for reducing the risk of being trapped in local minima and for increasing robustness to large anatomical variations. This has been demonstrated to be true in the registration of medical images (Hamm et al., 2009) and other real-world images (Munsell et al., 2009).

More recently, a novel groupwise registration framework, called Atlas Building by Self Organized Registration and Bundling, or ABSORB (Jia et al., 2010a, b), has been proposed for effective registration of a group of MR T1 images. Groupwise registration is achieved by registering each subject towards its selected neighbors, and thus the distribution of the whole dataset on the image space becomes more and more compact in a progressive manner. Pairwise registration, in ABSORB, is always performed between images that are close, and the problem caused by large deformation could be relieved to some extent. However, 
ABSORB is designed specifically for registration of scalar images, and the computation time is significantly longer than other pairwise registration methods (Jia et al., 2010a).

In this paper, we extend the intermediate template concept to work with pairwise DTI registration algorithms for improving the alignment accuracy and the robustness of diffusion tensor images. To this end, we first organize all images of the data group into a tree structure with similar images represented by connected nodes. The root node closest to the population center is selected as the template for registration. Each image will be registered with the help of intermediate templates determined along its own path with respect to the final template. The performance of the proposed groupwise DTI image registration algorithm is validated by experiments involving a real dataset and extensive comparisons with other methods, e.g., direct pairwise registration and ABSORB-based registration.

The rest of this paper is organized as follows. The proposed intermediate templates guided groupwise DTI registration framework is detailed in Section 2. In Section 3, extensive experiments for performance evaluation on real datasets, in comparison with other registration methods, are performed to demonstrate the registration accuracy and fiber alignment consistency. We conclude and discuss possible future extensions in Section 4.

\section{Method}

We detail in this section a novel intermediate templates guided DTI groupwise registration framework. The groupwise registration problem is solved with two steps: 1) compute the pairwise image similarity matrix, build a minimum spanning tree (MST), and determine the root node (which serves as the final template); and 2) register each image towards the final template with the help of intermediate templates distributed along its path to the root node. Registration between any two neighboring diffusion tensor images on the path can be performed using any conventional pairwise registration algorithm. For our case, we adopt two state-of-the-art techniques, TIMER (Yap et al., 2009c) and its fast version F-TIMER (Yap et al., 2010), for the pairwise registration. It is worth noting that other pairwise DTI registration methods can also be employed in this framework (Yang et al., 2008; Zhang et al., 2006).

\subsection{Image similarity}

For organizing the images based on their similarity, we first define a pairwise distance measure. Many different forms of similarity metrics between images have been defined in the literature (Alexander and Gee, 1999; Basser and Pierpaoli, 1996), including scalar-based metrics (e.g., metrics based on mean diffusivity or fractional anisotropy) and full-tensorbased metrics (Zhang et al., 2004). However, since we have adopted TIMER (Yap et al., 2009c) and its fast version F-TIMER (Yap et al., 2010) as our basic tools to do pairwise registration, it is essential to formulate the distance measure in a consistent way for both image pre-organization step and image registration step.

In TIMER, both volumetric information (e.g., the fractional anisotropy (FA) map) and edgerelated information (e.g., the edge magnitude extracted from the FA map and the tensor field) are involved in the distance measurement. However, when two images are not well aligned, especially in the early stage of the registration, including the edge information into the similarity measurement is not necessarily helpful. Hence, we opted to use FA, without edge information, in our formulation of distance measurement. FA (Basser and Pierpaoli, 1996) measures the degree of anisotropy of a tensor at location $x$ as

$F A(x)=\sqrt{\frac{3}{2}\left(\frac{\left(e_{1}-\bar{e}\right)^{2}+\left(e_{2}-\bar{e}\right)^{2}+\left(e_{3}-\bar{e}\right)^{2}}{e_{1}^{2}+e_{2}^{2}+e_{3}^{2}}\right)}$, where $e_{1}, e_{2}, e_{3}$, are the eigen-values of the 
corresponding tensor matrix and $\bar{e}$, is the mean eigen-value. For an isotropic medium, $F A=$ 0 , and for a fully anisotropic medium, $F A=1$. Its value is characteristically high for a voxel in the white matter (WM). But FA alone is not sufficient for fully capturing the difference between two images because of its poor discriminant power on gray matter (GM) and cerebrospinal fluid (CSF) (see Fig.1 (a) and (d)). For this reason, the apparent diffusion coefficient $(\mathrm{ADC})$ map $\left(A D C(x)=\left(e_{1}+e_{2}+e_{3}\right) / 3\right)$, that measures the average diffusivity in each voxel is also considered in distance measurement in addition to the FA map. The range of ADC values is dependent on the acquisition parameters. Some regions of the brain, such as the ventricles, contain mostly CSF, and thus ADCs typically have characteristically higher values (as shown in Fig. 1(b)) while other regions typically showing lower values. So ADC is a good measure for separating CSF and non-CSF tissue, while FA is helpful for distinguishing WM from non-WM tissues. By combining FA and ADC, the differences between two brain images in WM, GM and CSF can be fully accounted for, as indicated in Fig. 1 (c).

For each diffusion tensor image $S$, the corresponding FA and ADC maps are used to calculate the distance. To combine two scalar maps properly, it is necessary to normalize their values to have the same range. As the FA value has an inherently fixed range ([0, 1]), we only need to rescale all ADC values to be within $[0,1]$ by dividing each of them by the maximum ADC value. The maximum ADC value is calculated over all subjects in the groupwise registration scenario for a fair comparison in the later steps.

We define the distance (dissimilarity) between subject $S$ and $T$ as the Euclidean distances between the corresponding FA maps $S_{F A}$ and the rescaled ADC maps $S_{A D C}$,

$$
d(S, T)=\left(\sum_{p=1}^{M}\left(S_{F A}(p)-T_{F A}(p)\right)^{2}+\sum_{p=1}^{M}\left(S_{A D C}(p)-T_{A D C}(p)\right)^{2}\right)^{\frac{1}{2}},
$$

where $M$ is the total number of voxels in each subject, and $S_{F A}(p)$ and $S_{A D C}(p)$ are the value of the $p$-th voxel on the FA map and the rescaled ADC map of subject $S$, respectively. To better measure the distance between two neighboring subjects, after building a graph with each node representing a subject and each edge weighted with the above-mentioned distance, we go one step further to obtain the shortest distance defined on the $k$-NearestNeighbor $(k N N)$ graph with the Dijkstra algorithm (Dijkstra, 1959) to estimate the local structure. Thus, the distance between two images $S$ and $T$ can be updated as $d_{k}(S, T)$, which is the shortest distance between these images on the $k \mathrm{NN}$ graph. It is shown in our experiments that the above $k \mathrm{NN}$ graph based distance can help build a more balanced tree, which can effectively reduce the computing time of the registration.

\subsection{Registration path allocation and intermediate templates selection}

Based on the image dissimilarity measure defined above, we first build a fully connected graph with each node representing a subject and each edge being weighted by the distance, and then a tree can be extracted from the graph. In this way, all images can be organized into a tree structure where only similar images are connected. Here, the minimum spanning tree (MST) algorithm, which can be efficiently computed by using Kruskal's algorithm (Kruskal, 1956) is employed. The MST is constructed to be the tree with the minimum total edge length. However, the tree generated by Kruskal's algorithm does not have a specific root node, which in our case is important for determining the final template. To build a more balanced tree (Balakrishnan, 1997) and to reduce the average pairwise registration computation cost for each subject, the root node is selected to be the one with the minimum path length to all other images. A path length is defined as the number of segments involved 
in traversing a pathway between two nodes along the tree. The root node, considered as the population center, is used as the template in subsequent registration steps.

\subsection{Sequential registration}

After all images in the dataset have been organized into a tree, each subject is either directly connected to the root template or indirectly by one or more intermediate templates. We take a sequential approach for registering the entire population. For an image that is connected to the root template directly, any pairwise diffusion tensor image registration method can be employed and the registration result is guaranteed since these two images are generally very similar to each other. For images that are not directly connected to the template, registration is performed sequentially along the path determined during the tree-building process. The subject image is aligned to its first intermediate template (represented as its immediate parent node on the path), and then to its next intermediate templates one by one along the path to the root image. This scheme allows the images to be registered more accurately, especially for those images that are far away from the population center.

As an illustration, we construct the MST based on a real data set with 22 subjects, as shown in Fig. 2. In this MST, subject 02 (the square at the top level) is chosen as the root, or the final template. For the four subjects (represented by diamond nodes at the second level) which have direct connection to the root, their registration can be completed in one step with reasonable accuracy, since they are similar to the template. There are one or two intermediate templates selected for each of other subjects (represented by the circle or ellipse at the third or fourth level) to help guide its respective registration to the final template. It can also be observed that in our experiments, the minimum spanning tree (in Fig. 2) constructed with $k N N$ graph based distance measurement is more balanced with a smaller height 3 than the tree with height 5 if built directly with the distance defined in Eq. 1.

\subsection{Discussion}

The main advantages of our method are: 1) the final template, which is closest to the population center of the image space, is automatically selected to alleviate potential bias; 2) the whole registration process is guided by intermediate templates determined from the existing set of images, effectively decomposing the deformation process into a series of smaller ones which can be solved more efficiently and accurately.

This proposed method is well-suited for a large dataset. In a large dataset, in general, it is more likely that better intermediate templates can be found to guide the registration between any two images, especially for the two images with large deformation. The only concern might be the running time for the whole process, which is analyzed below for the individual steps involved: (1) the selection of affine template, (2) tree-building, and (3) intermediate templates guided registration. In the following discussions, we assume a total of $N$ DTI images in the dataset.

In the step (1) of affine template selection, only the pairwise affine registration between the FA maps is needed, and the complexity is $O\left(N^{2}\right)$. In our experiments, each pairwise affine registration takes less than 30 seconds. This can be further reduced if affine registrations are applied to down-sampled images. In the step (2) of MST building, since the distance measure is defined on the scalar maps (FA map and ADC map), the distance between two images can be computed very rapidly. The overall complexity for computing the pairwise distance matrix is $O\left(N^{2}\right)$. The running time of Kruskal's algorithm for MST construction is also very low (within seconds for $N=100$ ). The most computationally demanding step is the step (3) of sequential registrations, where each subject is registered towards the final 
template along its respective path onto the root node. TIMER will be applied once for each subject, giving a complexity of $O(N)$, and each run of TIMER takes approximately 0.5 hour. F-TIMER ( $\sim .15$ hour per run) is much faster than TIMER. The average runs of F-TIMER are directly related to the scale of the MST. Here, the scale of the tree is evaluated based on its height, which will determine the maximal number of intermediate templates, as well as the maximum runs of F-TIMER. In graph theory (Balakrishnan, 1997), a balanced tree will, in general, give the smallest height, i.e., $O(\log N)$ (refer Section 2.2). So the total runs of FTIMER is in the order of $O(N \log N)$, which is roughly linearly proportional to the size of the dataset. Thus we can see that the proposed groupwise registration framework can be feasibly extended to a larger dataset.

\section{Experiments}

We have performed a number of experiments to evaluate the accuracy of the proposed intermediate templates guided DTI groupwise registration algorithm. The dataset consists of a set of diffusion tensor images of real subjects with significant anatomical variation. We first demonstrate that our method can successfully learn the structure of the dataset, from which a tree can be constructed for identifying the root node and also the appropriate intermediate templates. Upon successful registration, we display the average images of the aligned images given by different registration methods for gauging registration accuracy both qualitatively and quantitatively. A fiber-tracking based experiment was also performed to gauge the effectiveness of the algorithm on registering white matter microstructures in brain.

\subsection{Data set and pre-processing}

Diffusion tensor images were acquired from 22 subjects using a $1.5 \mathrm{~T}$ scanner with 30 gradient directions and diffusion weighting $\mathrm{b}=700 \mathrm{~s} / \mathrm{mm}^{2}$. Imaging dimension is $256 \times 256 \times 70$ with a $240 \times 240 \mathrm{~mm}^{2}$ field of view (FOV) and a $0.9375 \times 0.9375 \times 2.5 \mathrm{~mm}^{3}$ image resolution.

All of the diffusion tensor images were skull-stripped to extract the brain parenchyma before they were used in the experiments. All images were first affinely aligned together for removing global shape differences, such as translation, rotation, scaling and shearing. We adopted the same tree-building strategy as proposed in this paper to select the root template image as the target of affine registration. The distance is calculated based on a pair of affine registered FA maps. For instance, for two DTI images $S$ and $T$, we can linearly register the FA map $S_{F A}$ to the FA map $T_{F A}$ to obtain the registered image $S_{F A}^{\prime}$. The intensity difference between $T_{F A}$ and $S_{F A}^{\prime}$ can be computed as follows:

$$
\operatorname{dif}\left(S_{F A}^{\prime}, T_{F A}\right)=\left(\sum_{p=1}^{M}\left(S_{F A}^{\prime}(p)-T_{F A}(p)\right)^{2}\right)^{\frac{1}{2}},
$$

where $M$ is the number of voxels. The distance between two images $s\left(S_{F A}, T_{F A}\right)$ is defined as follows to make it symmetric:

$$
s\left(S_{F A}, T_{F A}\right)=\frac{1}{2}\left[\operatorname{dif}\left(S_{F A}^{\prime}, T_{F A}\right)+\operatorname{dif}\left(T_{F A}^{\prime}, S_{F A}\right)\right] .
$$

Based on the distance defined in Eq. 3, we can build MST by applying the same method described in Section 2.2, and then the root image is chosen as the template for removing global differences of all images by linearly registering them to the selected template. 


\subsection{Different registration methods}

In our framework, we adopted two state-of-the-art DTI registration techniques, TIMER (Yap et al., 2009) and its fast version F-TIMER (Yap et al., 2010), to achieve the pairwise registration. To speed up registration, F-TIMER, due to its speed, was used for registering each subject image to its intermediate templates, while TIMER was employed in the last step for the final refinement of the non-rigid registration. TIMER uses a more localized Gaussian-based propagation of the driving voxel deformations. F-TIMER, on the other hand, uses the thin-plate splines (TPS) (Rohr et al., 2001; Wahba, 1990), which can effectively model global deformation although it might not be able to model fast spatiallyvarying deformations.

As the baseline for comparison, we performed a direct pairwise registration of each image to the same final template by TIMER with the same parameters and more iterations. For the proposed intermediate templates guided registration framework, the numbers of iterations in F-TIMER and TIMER are set to 8 and 20, respectively. In contrast, we set the iteration number to 30 for direct pairwise registration based on TIMER. The total computational time of the proposed framework was $\sim 14$ hours for 22 subjects, which is similar to that of direct pairwise registration that requires more iterative registration steps and thus needs $\sim 13$ hours. It is worth noting that the processing time reported here is based on single CPU. Using the intermediate templates for registration gives greater robustness to local minima. In contrast, direct pairwise registration is more susceptible to local minima, and not much improvement can be gained by further refinement. We also compared the proposed method with ABSORB (Jia et al., 2010a). Specifically, we use ABSORB on the FA maps of the DTI images to estimate a set of initial deformations, followed by a registration refinement via TIMER to obtain the final deformation field.

\subsection{Results}

Tree Structure, Intermediate Templates, and Final Template-One advantage of the proposed registration algorithm is that several subjects are selected to serve as the intermediate templates to bridge two subjects together for a guided registration. An example is given in Fig. 3. The relatively large differences between a subject $S$ and the template $T$ makes it difficult to register them directly. With the help of intermediate templates, $I T_{1}$ and $I T_{2}$, the registration from subject $S$ to $T$ could be solved more easily by following the path $S \rightarrow I T_{1} \rightarrow I T_{2} \rightarrow T$, since the difference between any two successive subjects, both inspected visually and measured by the dissimilarity measure defined in Section 2, is much smaller than that between $S$ and $T$ directly.

Fig. 4 shows that all images in the dataset can be organized into a single tree structure by employing the minimum spanning tree algorithm with the help of the pairwise dissimilarity measure defined in Section 2. The root subject is taken as the final template and placed at Level 1. Totally, 4 subjects are quite similar to the template, and thus they are placed at Level 2 with direct connections to the template. So the registration of subjects in Level 2 can be performed using conventional pairwise registration easily. Images at Level 3 or 4 are connected to the template indirectly through the intermediate templates. As we will show later in this paper, the subjects in the lower levels of tree generally benefit more from the current registration framework.

Registration Accuracy - Qualitative Assessment-We now demonstrate that the registration accuracy can be improved with the help of intermediate templates. Fig. 5 shows the registration results of different methods, where the subject image (with three different consecutive slices shown in (d) (f)) is registered to the template (a). The red crosses are placed as landmarks of corresponding positions in (a) the template image, (b) the warped 
image after the proposed intermediate templates guided registration, and (c) the warped image after direct pairwise registration. We can see that the proposed method can achieve higher registration accuracy as it can better align the white matter structures (indicated by high FA values) in the subject in correspondence to the template. Similar observation can be made in the circled area. To further demonstrate that the branching structure appearing in the circled region of (b) is indeed a real brain structure, three neighboring slices are displayed in Fig. 5(d)(e)(f). Based on this example, we can conclude that the intermediate templates are conducive to a more accurate registration.

Registration Accuracy - Quantitative Assessment-To further demonstrate the registration accuracy of the proposed algorithm at a group level, we evaluated the registration consistency by measuring the pairwise distances of the warped images. For each warped image, we computed its FA and ADC maps. For FA (or ADC) map of each subject pair, the pairwise distance was defined as the mean of the sum of the squared error (MSSE) calculated voxel-wisely over the whole image space. The average pairwise distance over all registered image pairs is reported in Table 1. It is obvious that the proposed method results in more consistent alignment of images in the population. For both ADC and FA maps, the proposed intermediate templates guided registration method can generate a more compact and better aligned image set than the direct pairwise registration (with $\boldsymbol{p}=\mathbf{0 . 0 1}$ and $\boldsymbol{p}=$ $\mathbf{0 . 0 0 5}$, respectively). It also can be seen that the ABSORB-based registration framework has an improved result to the direct pairwise registration algorithm, but it is still worse than the proposed method. The possible reason is that the deformation fields obtained by the groupwise registration on FA maps cannot provide a good initialization to the further TIMER-based refinement as there are not many structural details in FA map, especially for the cortical regions.

Atlas Building-An atlas can be constructed from the underlying dataset by averaging the aligned images. An atlas as such can give us a direct and intuitive means of inspecting the registration accuracy. A sharper atlas indicates better registration accuracy in contrast to a fuzzy atlas due to misaligned structures. The atlases built with affine registration, direct pairwise registration with TIMER and the proposed method are given in the left, middle, and right column of Fig. 6, respectively. We can observe that the proposed method gives a sharper atlas, especially in the cortical regions.

Comparison of Deformation Fields-Higher registration accuracy can sometimes be achieved by sacrificing the smoothness of the deformation field. In TIMER or F-TIMER, the cost function is regularized by a deformation-smoothness constraint. To demonstrate that the improvement of registration accuracy was indeed the result of employing intermediate templates, we computed the Jacobians and the values of bending energies (Rohr et al., 2001) on the position of each voxel based on the deformation fields estimated by different registration methods. Measurements used include the maximum Jacobian $\left(\mathrm{J}_{\max }\right)$, and the maximum and mean bending energy values $\left(\mathrm{BE}_{\max }\right.$ and $\left.\mathrm{BE}_{\text {mean }}\right)$. Specifically, we use $\mathrm{BE}_{\mathrm{max}, \mathrm{i}}$ to denote the maximum bending energy value calculated from the deformation field for warping subject $i$ to the template, and $\mathbf{J}_{\max , \mathbf{i}}$ for its corresponding maximum Jacobian. The mean and maximum values of each measurement over all the 22 subjects are given in Table 2. We can see that the proposed method almost always gives smaller Jacobians or bending energy value, except the maximum Jacobian. That is, the proposed registration framework can achieve better registration accuracy with comparable or even slightly smoother deformation fields. The $p$-values of the pairwise $t$-test on the corresponding values are also shown in Table 2 to reinforce the same conclusion.

The distributions of Jacobians on the deformation field generated by two registration methods are shown in Fig. 7 (a) and (b), respectively. The mean Jacobians for the proposed 
method is 0.96 , which is closer to 1 than that of the direct pairwise registration method (0.93). The standard deviation of Jacobians of the proposed method is 0.103 , which is smaller than that of the direct method (0.109). This implies that the Jacobians given by our method are more concentrated around 1, indicating smoother deformation fields. We also illustrate the sample deformation fields and bending energy value maps from different methods in Fig. 8. It can be observed that the proposed method can, in general, generate smoother deformation fields.

Alignment of Fiber Bundles-To evaluate the effectiveness of the proposed algorithm in aligning the brain microstructures, a fiber tracking based experiment was performed, where the fiber tracts from each subject are warped to the template space for gauging consistency (Gerig et al., 2004; Jiang et al., 2006).

To demonstrate the fiber registration results, we placed three different ROIs in the region of genu, splenium and the body of the corpus callosum (CC) in the template space (as shown in Fig. 9) so that fiber bundles passing through these ROIs could be extracted, i.e., using DTI studio (Jiang et al., 2006), for comparison. Each ROI in the template space was first warped back to the individual subject image space using the inverse of the estimated deformation field. Then fiber tracking was performed on each individual subject based on the warped ROI. The tracked fiber bundles were finally warped to the common template space using those estimated deformation fields. In DTI studio, the FA start threshold was set to 0.25 , the FA stop threshold to 0.2 , and the stop turning angle to $70^{\circ}$. The same tracking parameter and procedure were applied to all images, including the template. The distance between two fiber bundles was then evaluated using the distance measure:

$$
\frac{1}{|\mathscr{F}|+|\mathscr{G}|}\left[\sum_{F_{i} \in \mathscr{F}} \min _{G_{j} \in \mathscr{G}} d\left(F_{i}, G_{j}\right)+\sum_{G_{j} \in \mathscr{G}_{i}} \min _{F_{i} \in \mathscr{F}} d\left(F_{i}, G_{j}\right)\right],
$$

where $\boldsymbol{d}\left(\boldsymbol{F}_{\boldsymbol{i}}, \boldsymbol{G}_{j}\right)$ is a pairwise distance measurement between each fiber pair and $\boldsymbol{F}_{\boldsymbol{i}} \in \boldsymbol{z}$ and $\boldsymbol{G}_{\boldsymbol{j}} \in \mathscr{G}$ which is defined as the mean of the closest distance for every point pair of two fibers. Here, 7 and $\mathscr{G}$ are the fiber bundles tracked in each subject, respectively. This measurement can be used as indication of fiber alignment and two perfectly aligned fibers give a distance of zero.

Pairwise distances between the warped subject fibers and the original template fibers are tabulated in Table 3. Recall that our built tree consists of 4 levels. In Table 3, we focus on the fiber bundle registration results of the images at the third and fourth levels, since the registration of these images are guided by intermediate templates.

The results in Table 3 indicate that the proposed intermediate templates guided registration gives an average of $20 \%$ smaller fiber misalignment error. To further demonstrate the benefit brought by the intermediate templates, we break down the results according to different levels of the tree. For the 10 subjects at the third level, the average decrease in fiber misalignment error is $1.24 \mathrm{~mm}$ (20.03\%); while for the 7 subjects at the fourth level the decrease is $1.20 \mathrm{~mm}(21.34 \%)$. This indicates that images further down the tree benefit more from the utilization of intermediate templates. The distributions of the point-wise fiber distances to the fibers in the template are shown in Fig. 10, which clearly indicates the improvement obtained by the use of intermediate templates.

To illustrate the fiber bundles alignment results more clearly, one subject is selected to show the different aligned results to the template by the direct registration and the proposed registration method, respectively. In Fig. 11, two fiber bundles, genu (Fig. 10a) and 
splenium (Fig. 10b) of the corpus of callosum, tracked in the template are overlaid with the corresponding warped subject fiber bundles. In each plot, the distance-weighted colorcoding result on the corresponding fiber bundles in the template is shown in the top row, and the actual alignment results are shown in the bottom row, with red indicating the template bundle and green for subject bundle. By visual inspection, we can see that the proposed method (left column) gives a more consistent alignment of the fiber bundles than direct pairwise registration (right column).

To visualize the fiber tracking results on the mean image, the same two ROIs defined above were used to track the fiber bundles in the mean diffusion tensor images computed from the registered images. The results are given in Table 4. In general, the mean image given by the proposed algorithm gives a denser fiber bundle with more fibers. There is also an increase in the maximum and minimum fiber lengths, albeit a slight decrease in the average fiber length. If we select the longest 300 fibers in each extracted bundle, the average fiber length given by the proposed algorithm is much longer than the results from other methods. This also indicates that better registration allows better alignment of fibers from different subjects.

\section{Conclusions}

A new method has been proposed for more accurate and robust group-wise registration of diffusion tensor images. Based on the dissimilarity measure defined based on the combination of the FA and ADC maps, all DT images are organized into a minimum spanning tree where only similar images are directly connected. The image closest to the population center is chosen as the final template. For subject images far from the template, one or more intermediate templates will be utilized to guide their registration. Registration of any two connected images can be achieved with relative ease since they are similar in shape. Registration accuracy and robustness has been demonstrated using a set of real diffusion tensor images. Fiber misalignment error is found to be significantly reduced compared to direct pairwise registration. In future, we will examine the scalability of the proposed framework and test it on large clinical datasets with white matter disorders to evaluate its performance in aiding the detection of disease-related abnormalities.

\section{References}

Alexander, DC. Visualization and Processing of Tensor Fields. Berlin Heidelberg: Springer; 2006. p. 83-106.

Alexander DC, Gee JC. Elastic matching of diffusion tensor images. Computer Vision and Image Understanding. 1999; 77:233-250.

Balakrishnan, VK. Graph Theory. 1st ed.. McGraw-Hill; 1997.

Baloch S, Davatzikos C. Morphological appearance manifolds in computational anatomy: Groupwise registration and morphological analysis. NeuroImage. 2009; 45:S73-S85. [PubMed: 19061962]

Baloch, S.; Verma, R.; Davatzikos, C. Information Processing in Medical Imaging (IPMI), 2007. Netherlands: 2007. An Anatomical Equivalence Class based Joint Transformation-Residual Descriptor for Morphological Analysis; p. 594-606.

Barmpoutis A, Vemuri BC. Groupwise registration and atlas construction of 4th-order tensor fields using the R+ Riemannian metric. MICCAI. 2009:640-647.

Basser PJ, Pierpaoli C. Microstructural and physiological features of tissues elucidated by quantitativediffusion-tensor MRI. Journal of Magnetic Resonance, Series B. 1996; 111:209-219. [PubMed: 8661285]

Bihan DL, Mangin J-F, Poupon C, Clark CA, Pappata S, Molko N, Chabriat H. Diffusion tensor imaging: concepts and applications. Journal of Magnetic Resonance Imaging. 2001; 13:534-546. [PubMed: 11276097] 
Dijkstra EW. A note on two problems in connexion with graphs. Numerische Mathematik. 1959; $1: 269-271$.

Gerig, G.; Gouttard, S.; Corouge, I. Analysis of brain white matter via fiber tract modeling; Proceedings of the IEEE EMBS; 2004. p. 4421-4424.

Hamm, J.; Davatzikos, C.; Verma, R. MICCAI 2009. London, UK: 2009. Efficient large deformation registration via geodesics on a learned manifold of images.

Huang H, Zhang J, Wakana S, Zhang W, Ren T, Richards LJ, Yarowsky P, Donohue P, Graham E, van Zijl PCM, Mori S. White and gray matter development in human fetal, newborn and pediatric brains. NeuroImage. 2006; 33:27-38. [PubMed: 16905335]

Jia H, Wu G, Wang Q, Shen D. ABSORB: Atlas Building by Self-Organized Registration and Bundling. NeuroImage. 2010a; 51:1057-1070. [PubMed: 20226255]

Jia H, Wu G, Wang Q, Shen D. ABSORB: Atlas Building by Self-Organized Registration and Bundling. CVPR 2010. 2010b

Jiang H, van Zijl PC, Kim J, Pearlson GD, Mori S. DtiStudio: Resource program for diffusion tensor computation and fiber bundle tracking. Computer Methods and Programs in Biomedicine. 2006; 81:106-116. [PubMed: 16413083]

Joshi S, Davis B, Jomier M, Gerig G. Unbiased diffeomorphic atlas construction for computational anatomy. NeuroImage. 2004; 23 Suppl 1:S151-S160. [PubMed: 15501084]

Kim, M-J.; Kim, M-H.; Shen, D. Learning-based Deformation Estimation for Fast Non-rigid Registration. IEEE Workshop on Mathematical Methods in Biomedical Image Analysis (MMBIA); Anchorage, Alaska. 2008.

Kruskal JB. On the Shortest Spanning Subtree of a Graph and the Traveling Salesman Problem. Proceedings of the American Mathematical Society. 1956; 7:48-50.

Lee AD, Lepore N, Barysheva M, Chou YY, Brun CA, Madsen SK, K.L M, de Zubicaray GI, Meredith M, Wright MJ, Toga AW, Thompson PM. Comparison of Fractional and Geodesic Anisotropy in Diffusion Tensor Images of 90 Monozygotic and Dizygotic Twins. ISBI'2008. 2008

Lepore N, Brun C, Pennec X, Chou Y-y, Lopez OL, Aizenstein HJ, Becker JT, Toga AW, Thompson PM. Mean Template for Tensor-Based Morphometry using Deformation Tensors. MICCAI 2007. 2007

Liu AK, Marcus KJ, Fischl B, Grant PE, Poussaint TY, Rivkin MJ, Davis P, Tarbell NJ, Yock TI. Changes in cerebral cortex of children treated for medulloblastoma. Int J Radiat Oncol Biol Phys. 2007; 68:992-998. [PubMed: 17379433]

Munsell, BC.; Temlyakov, A.; Wang, S. Fast Multiple Shape Correspondence by Pre-Organizing Shape Instances; IEEE Conference on CVPR; 2009. p. 840-847.

Pierpaoli C, Jezzard P, Basser JP, Barnett A, Chiro GD. Diffusion tensor MR imaging of human brain. Radiology. 1996; 201:637-648. [PubMed: 8939209]

Rohr K, Stiehl HS, Sprengel R, Buzug TM, Weese J, Kuhn MH. Landmark-Based Elastic Registration Using Approximating Thin-Plate Splines. IEEE Transactions on Medical Imaging. 2001; 20

Shen D, Davatzikos C. HAMMER: Heirarchical Attribute Matching Mechanism for Elastic Registration. IEEE Transactions on Medical Imaging. 2002; 21:1421-1439. [PubMed: 12575879]

Tang S, Fan Y, Shen D. RABBIT: rapid alignment of brains by building intermediate templates. NeuroImage. 2009

Wahba, G. Soc. Ind. Appl. Math. Philadelphia, PA: 1990. Spline models for observational data.

Yang J, Shen D, Davatzikos C, Verma R. Diffusion Tensor Image Registration Using Tensor Geometry and Orientation Features. MICCAI'08. 2008:905-913.

Yap, P-T.; Wu, G.; Zhu, H.; Lin, W.; Shen, D. MICCAI09. London, U.K: 2009a. Fast Tensor Image Morphing for Elastic Registration.

Yap P-T, Wu G, Zhu H, Lin W, Shen D. TIMER: Tensor Image Morphing for Elastic Registration. MMBIA. 2009b

Yap P-T, Wu G, Zhu H, Lin W, Shen D. TIMER: Tensor Image Morphing for Elastic Registration. NeuroImage. 2009c; 47:549-563. [PubMed: 19398022]

Yap P-T, Wu G, Zhu H, Lin W, Shen D. F-TIMER: Fast Tensor Image Morphing for Elastic Registration. IEEE Transactions on Medical Imaging. 2010 
Zhang H, Avants BB, Yushkevich PA, Woo JH, Wang S, McCluskey LF, Elman LB, Melhem ER, Gee JC. High-Dimensional Spatial Normalization of Diffusion Tensor Images Improves the Detection of White Matter Differences: An Example Study Using Amyotrophic Lateral Sclerosis. IEEE Transactions on Medical Imaging. 2007; 26:1585-1597. [PubMed: 18041273]

Zhang H, Yushkevich PA, Alexander DC, Gee JC. Deformable registration of diffusion tensor MR images with explicit orientation optimization. Medical Image Analysis. 2006; 10:764-785. [PubMed: 16899392]

Zhang H, Yushkevich PA, Gee JC. Registration of Diffusion Tensor Images. CVPR. 2004:842-847.

Zöllei L, Stevens A, Huber K, Kakunoori S, Fischl B. Improved tractography alignment using combined volumetric and surface registration. NeuroImage. 2010; 51:206-213. [PubMed: 20153833] 


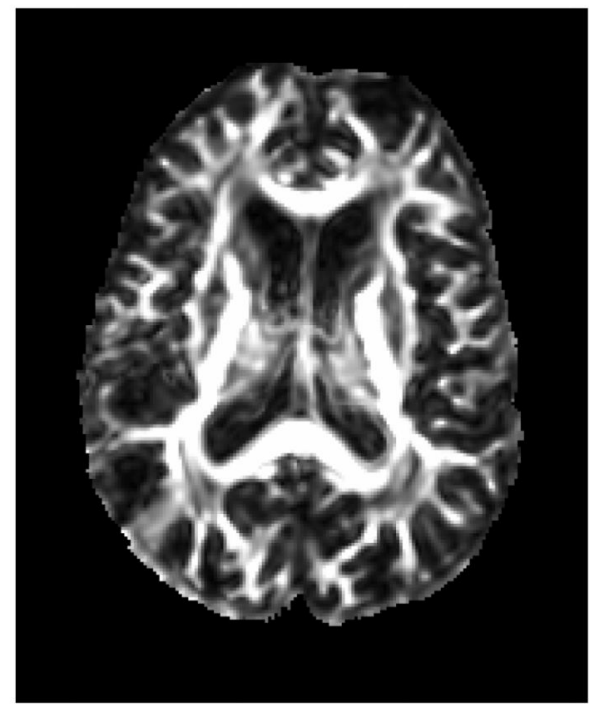

(a)

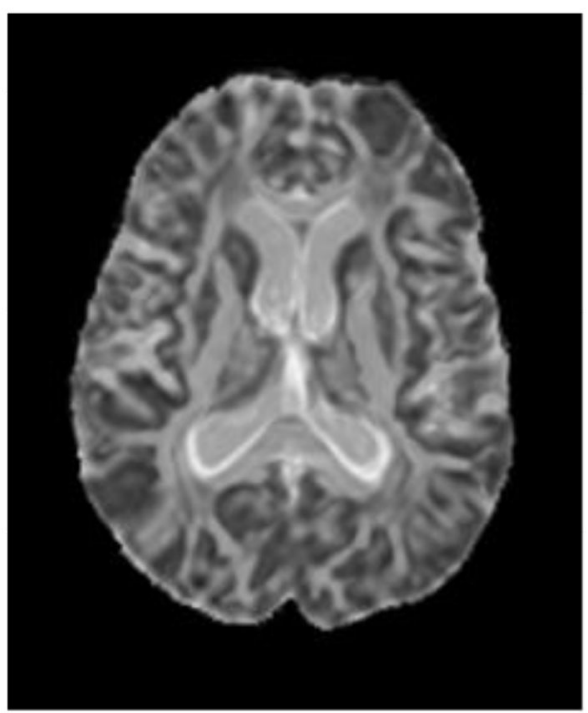

(c)

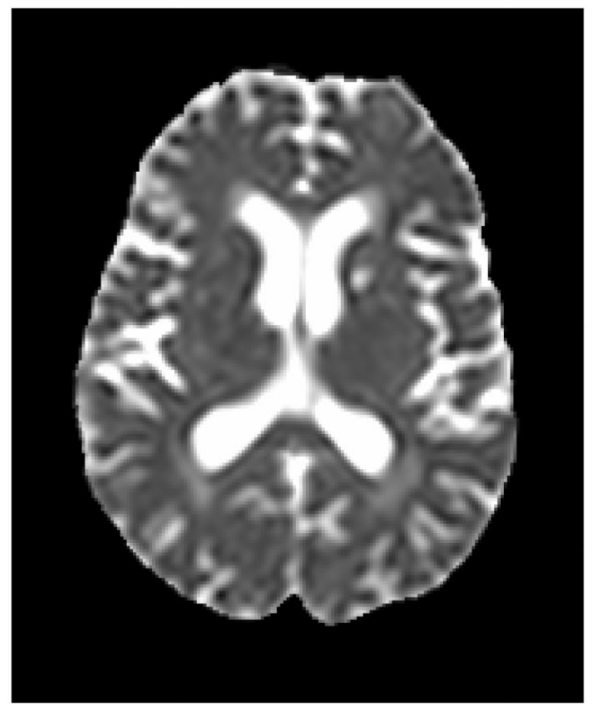

(b)

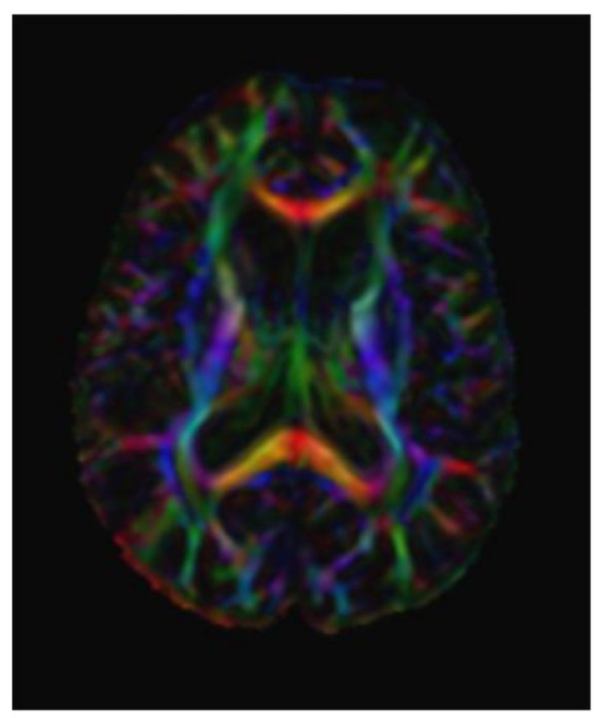

(d)

Fig. 1.

Two typical scalar maps of the diffusion tensor image: (a) the FA map and (b) the ADC map. These two maps are illustrated together with their combination map in (c). In (d), the color-coded representation of the FA-weighted first principal directions is shown: green for the anterior-posterior direction, blue for the superior-inferior direction, and red for the leftright direction. 


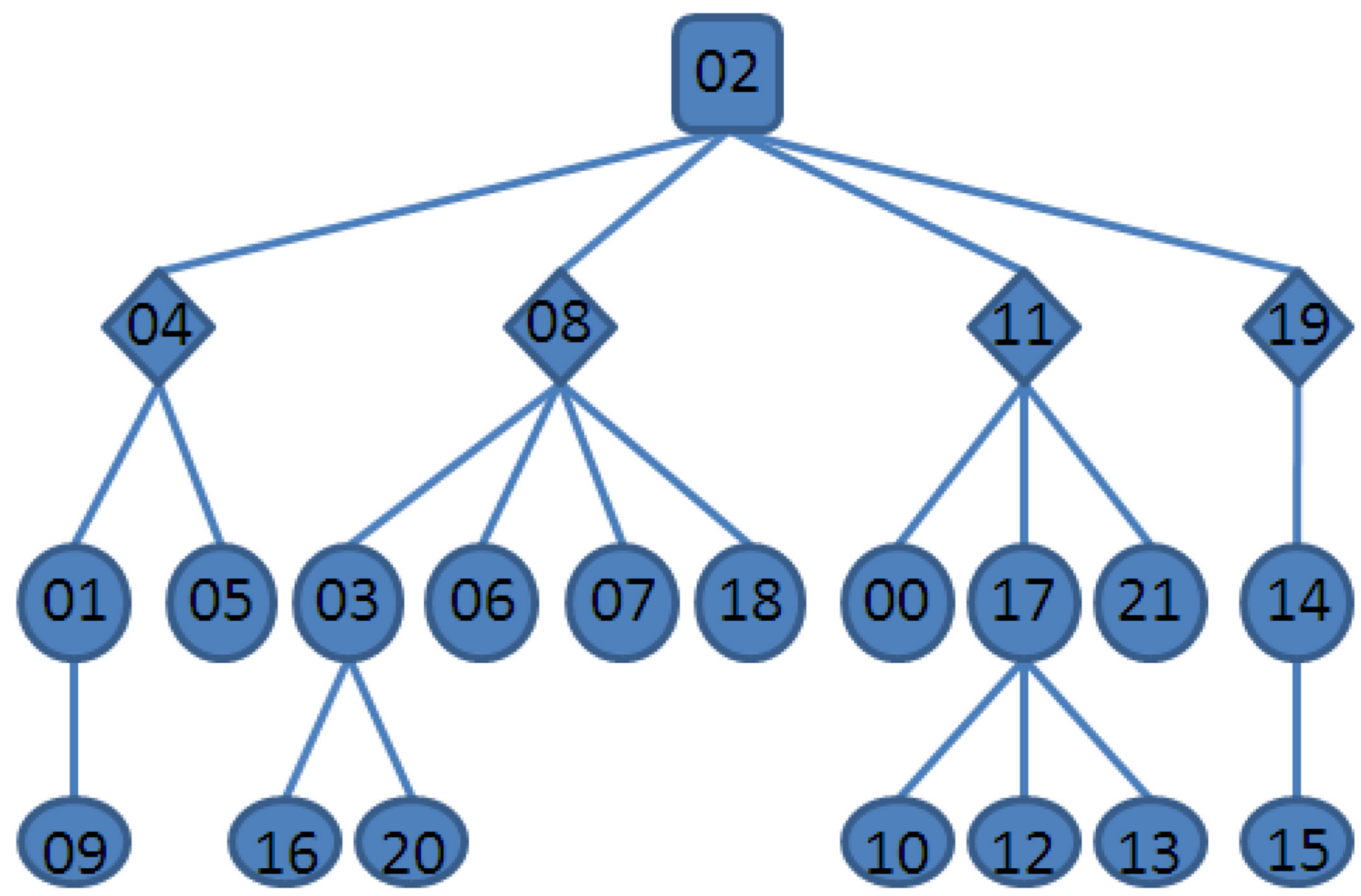

Fig. 2.

The minimum spanning tree constructed from the DTI dataset. Pairwise similarity is defined as the summation of Euclidean distances of the corresponding FA and scaled ADC maps, and the root node is selected to be the node with the minimum path length to all other nodes. 


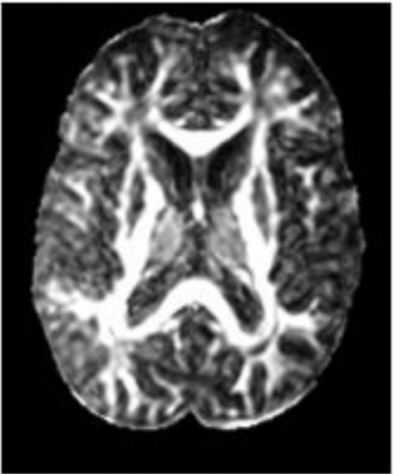

(a) Subject $S$

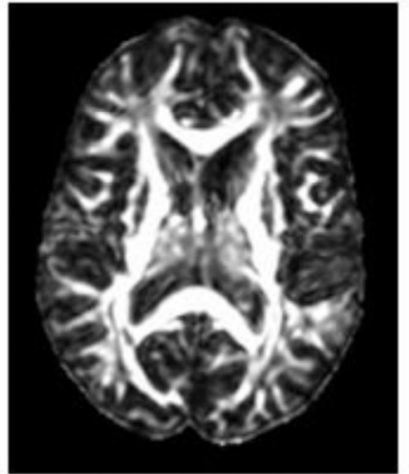

(b) $I T_{1}$

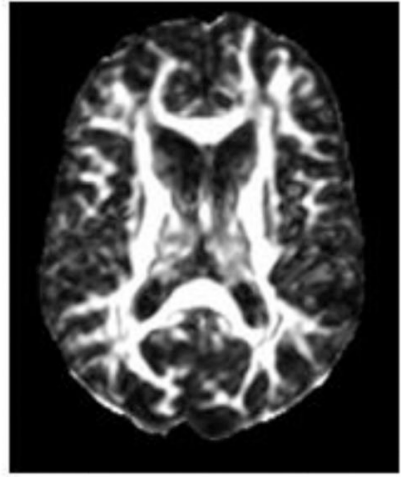

(c) $I T_{2}$

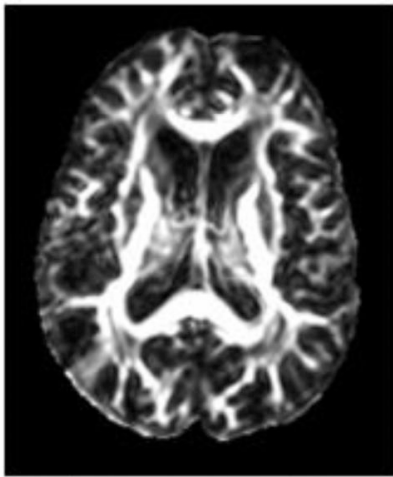

(d) Template $T$

Fig. 3.

Two intermediate templates $\left(I T_{1}\right.$ and $\left.I T_{2}\right)$ are selected by the proposed algorithm from the same data set to guide the registration between subject $S$ and template $T$, whose structures are significantly different from each other. In this way, the large deformation between subject $S$ and template $T$ could be decomposed into a series of smaller ones, which can be solved more easily and accurately by the existing pairwise DTI registration techniques. 


\section{图 图

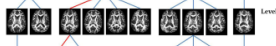

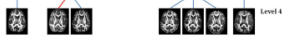

Fig. 4.

The tree structure constructed to represent the whole dataset. Registration of images at Level 3 and 4 are guided by the intermediate templates along their respective paths. Note that the images shown in Fig. 3 are connected by the red lines in this tree. 


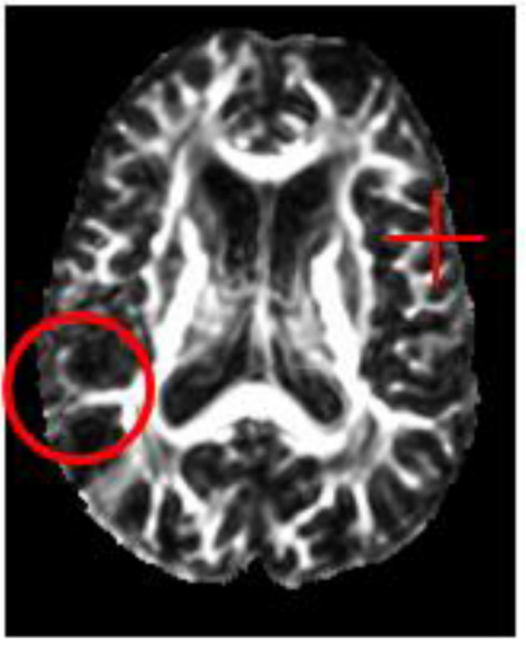

(a)

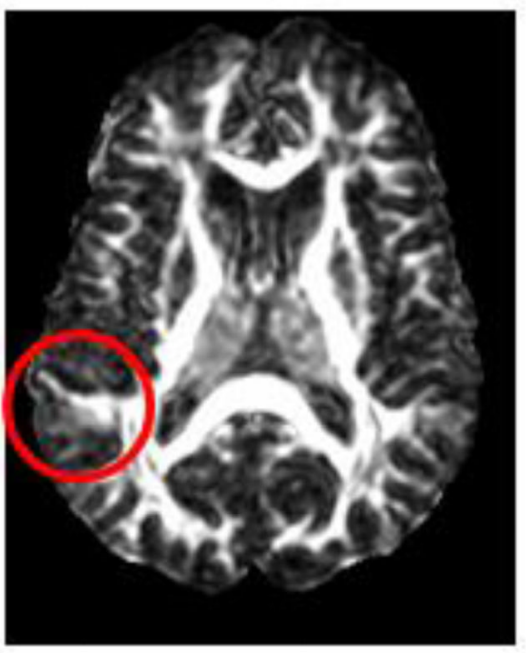

(d)

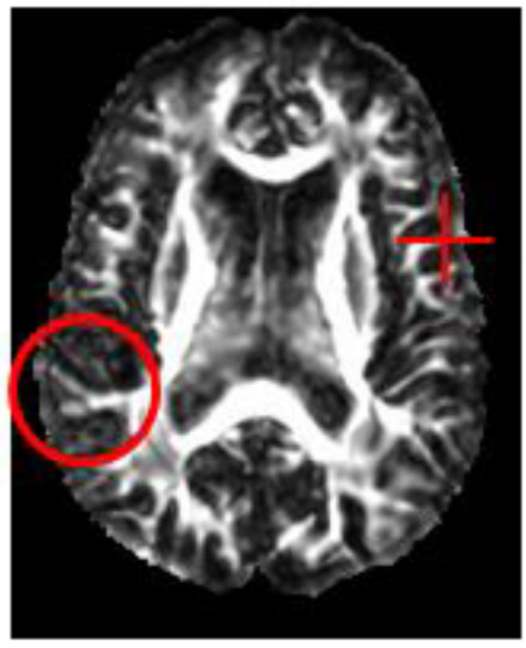

(b)

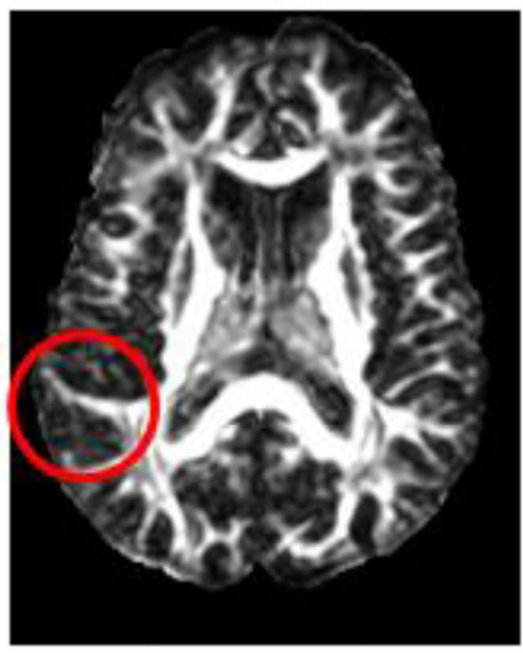

(e)

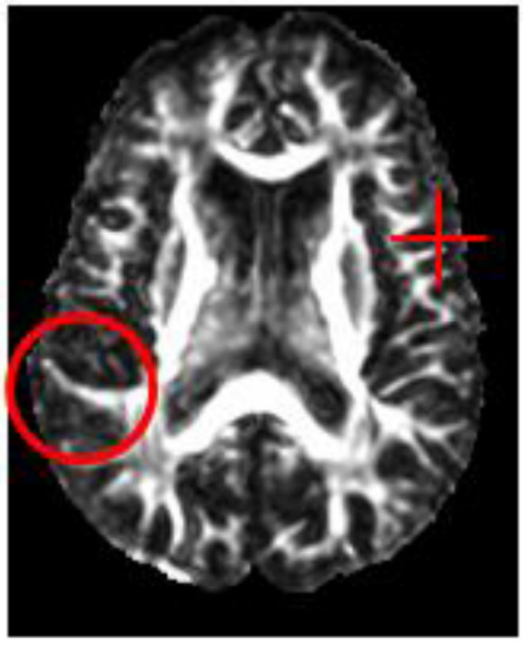

(c)

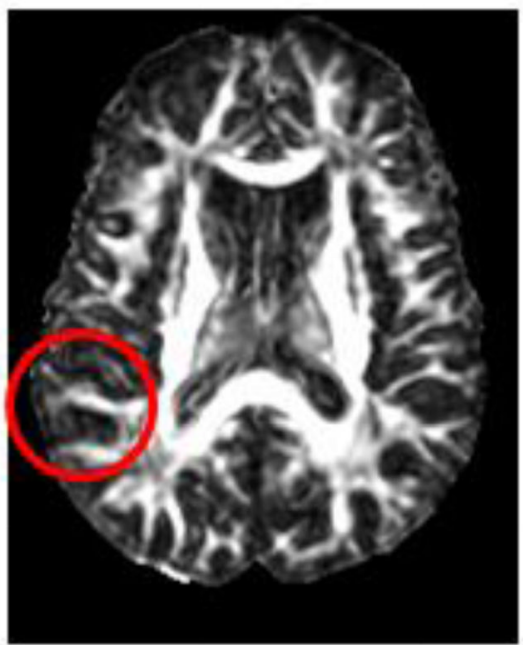

(f)

Fig. 5.

Comparison of registration accuracy between the proposed intermediate templates guided registration method and the direct pairwise registration algorithm. FA maps are shown for the same slice in (a) the template, (b) the image warped by the proposed method, and (c) the image warped by direct pairwise registration. The red crosses in (a) (c) indicate the same physical location in the different images. It can be seen that direct pairwise registration fails in some cortical regions, while the proposed method clearly demonstrates better registration accuracy. Similarly, the circled areas also indicate that the proposed groupwise registration method can obtain better correspondences than the direct registration. The corresponding slice in the original subject image is shown in (e) together with its two neighboring slices in 
(d) and (f). The proposed method successfully registers the branching structure that exists in both subject (f) and template (a), although it appears in different slices. 


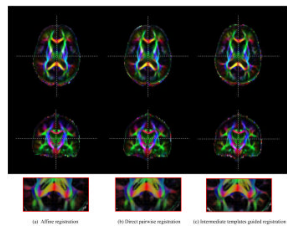

Fig. 6.

Atlases constructed from 22 subjects with affine registration (left column), direct pairwise registration (middle column) and the proposed intermediate templates guided registration method (right column). The FA weighted first principal directions are shown in their color coded representations: green for the anterior-posterior direction, blue for the superiorinferior direction, and red for the left-right direction. 


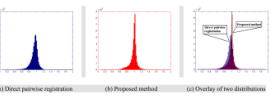

Fig. 7.

The distribution of Jacobians on the deformation field generated by (a) the direct pairwise registration and (b) the proposed intermediate templates guided method. The overlay of two distributions shown in (c) indicates that the distribution of the proposed method is more concentrated around 1 than the direct pairwise registration. 

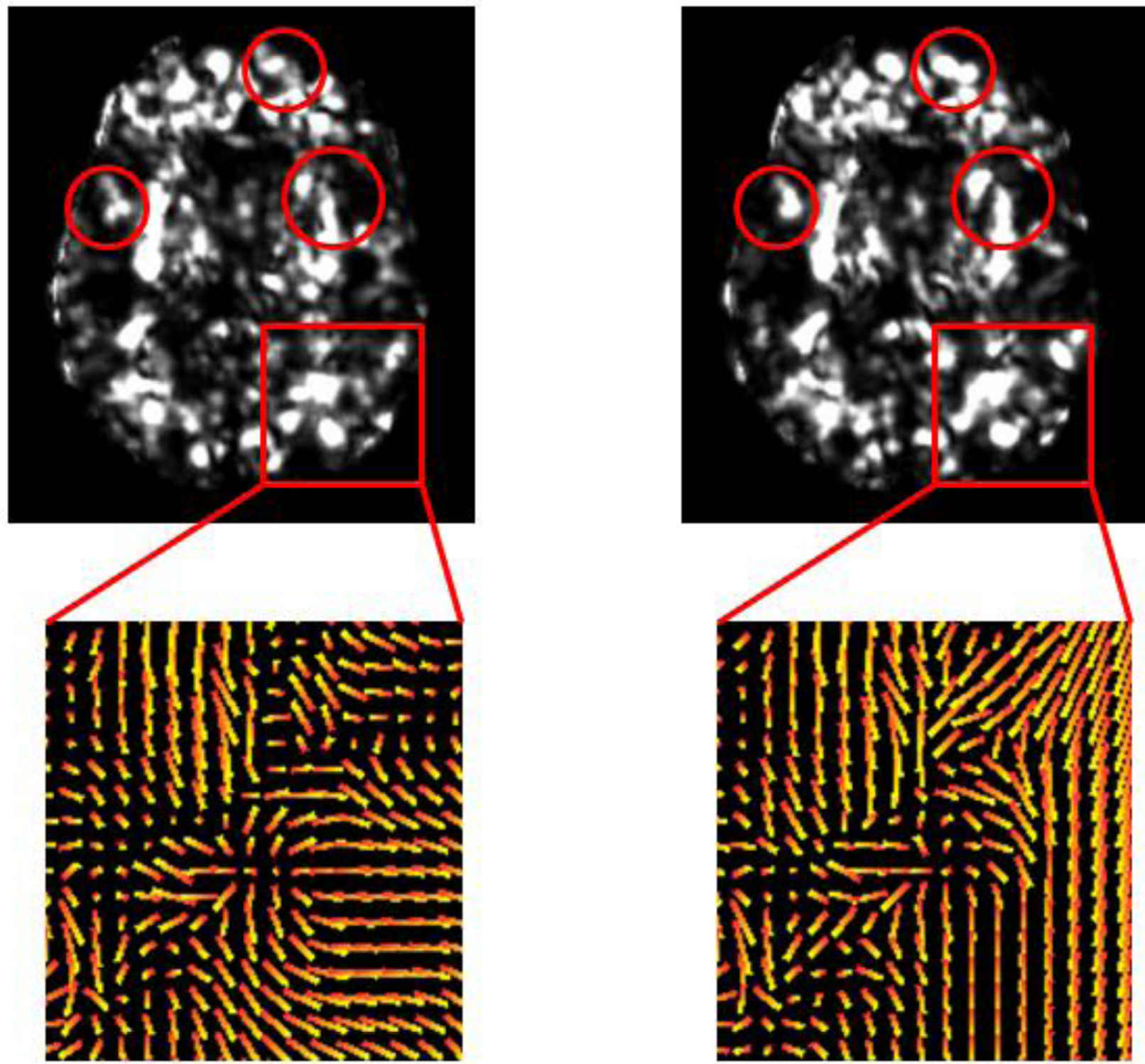

(a) Intermediate templates guided registration

(b) Direct pairwise registration

Fig. 8.

Bending energy value maps for (a) the intermediate templates guided registration, and (b) the direct pairwise registration. The brighter region indicates higher bending energy. For most brain regions, especially those in red circles, the proposed intermediate templates guided registration method can yield smoother deformation field and smaller bending energy. This is further confirmed by the close-up views of the vector fields. 


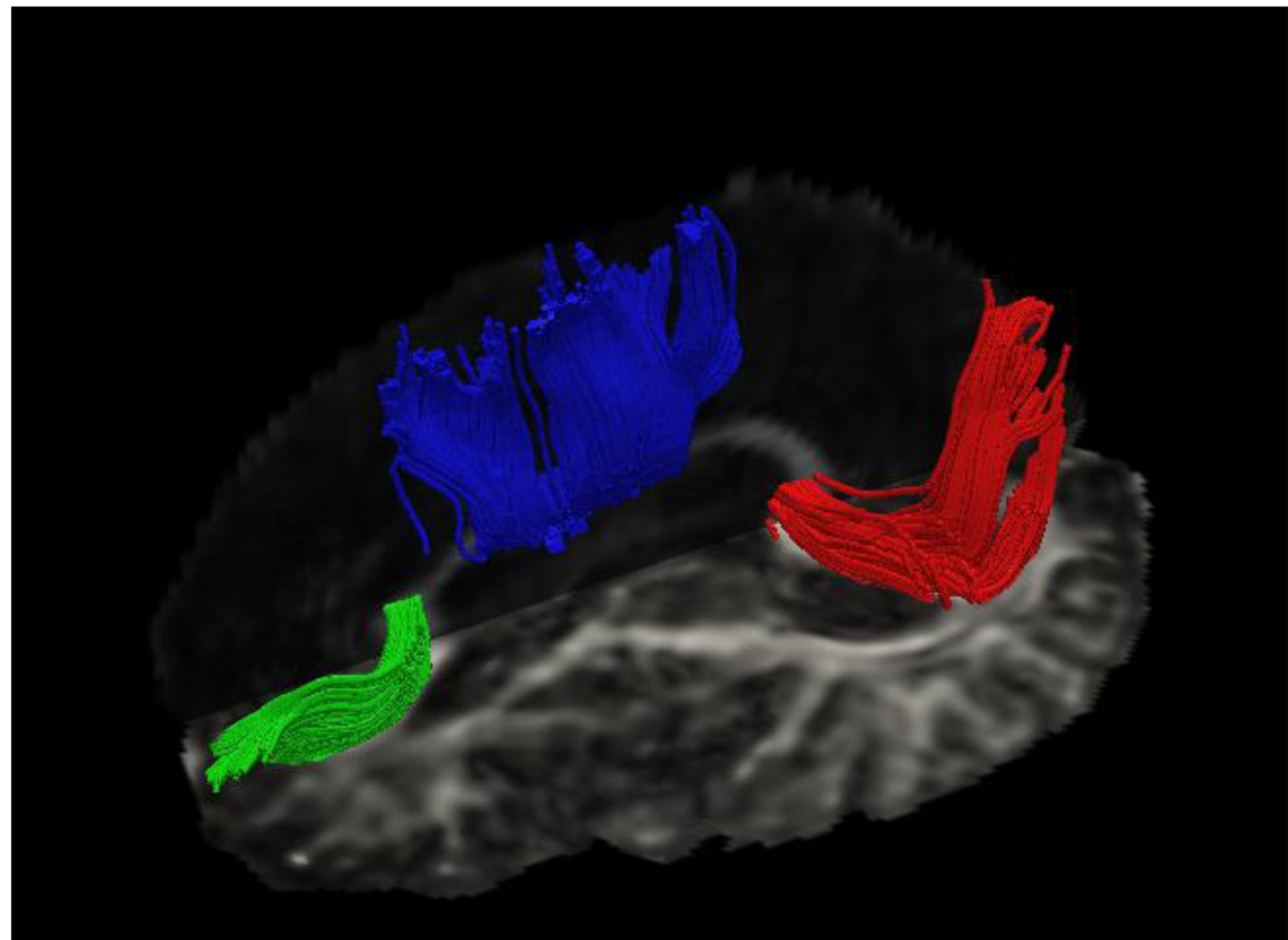

Fig. 9.

The illustration of fiber bundles used for evaluation. Shown in green, blue, and red are the fiber bundles in the genu, body and splenium of corpus callosum, respectively. 


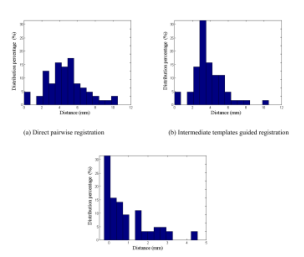

Fig. 10 .

The distributions of the point-wise fiber distances given by (a) the direct pairwise registration method, and (b) the proposed intermediate templates guided groupwise registration method. The distribution of the misalignment error reduction is provided in (c). 


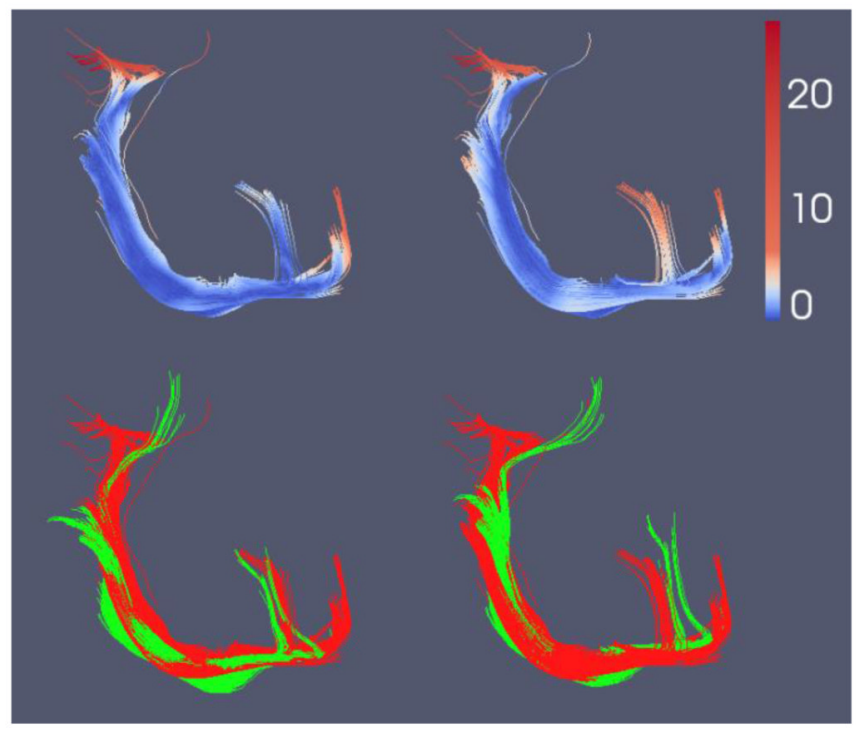

(a)

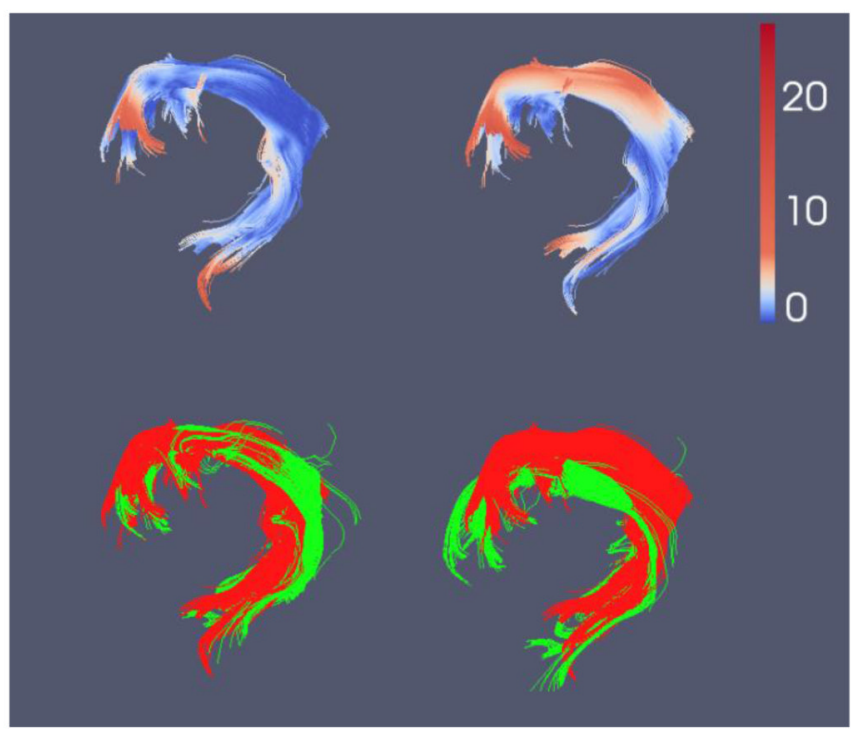

(b)

Fig. 11.

Accuracy of fiber bundle alignment. In each plot, the distance-weighted color-coding result on the corresponding fiber bundles in the template is shown in the top row, and the actual alignment results are shown in the bottom row. Red indicates the template fiber bundles, i.e., genu in (a) and splenium in (b), while green indicates the warped subject fiber bundles. The proposed method (left column) gives better fiber alignment than direct pairwise registration (right column). 


\section{Table 1}

The mean values of the pairwise Euclidean distances between all image pairs after the registration using different methods. The distance is calculated based on FA maps, ADC maps and the combined maps (as defined in Eq. 1). Note that the results based on the combination maps are obtained after normalization of the ADC map.

\begin{tabular}{|c|c|c|c|c|}
\hline & Affine registration & $\begin{array}{c}\text { Direct pairwise } \\
\text { registration }\end{array}$ & $\begin{array}{c}\text { ABSORB-based } \\
\text { registration }\end{array}$ & $\begin{array}{c}\text { Intermediate } \\
\text { templates guided } \\
\text { registration }\end{array}$ \\
\hline ADC & $10.9 \times 10^{-4}$ & $9.09 \times 10^{-4}$ & $8.87 \times 10^{-4}$ & $\mathbf{8 . 5 6 \times 1 0 ^ { - 4 }}$ \\
\hline FA & $6.32 \times 10^{-3}$ & $5.72 \times 10^{-3}$ & $5.63 \times 10^{-3}$ & $\mathbf{5 . 2 4 \times 1 0 ^ { - 3 }}$ \\
\hline FA+ADC & $8.42 \times 10^{-3}$ & $7.49 \times 10^{-3}$ & $7.43 \times 10^{-4}$ & $\mathbf{7 . 2 2 \times 1 0 ^ { - 3 }}$ \\
\hline
\end{tabular}




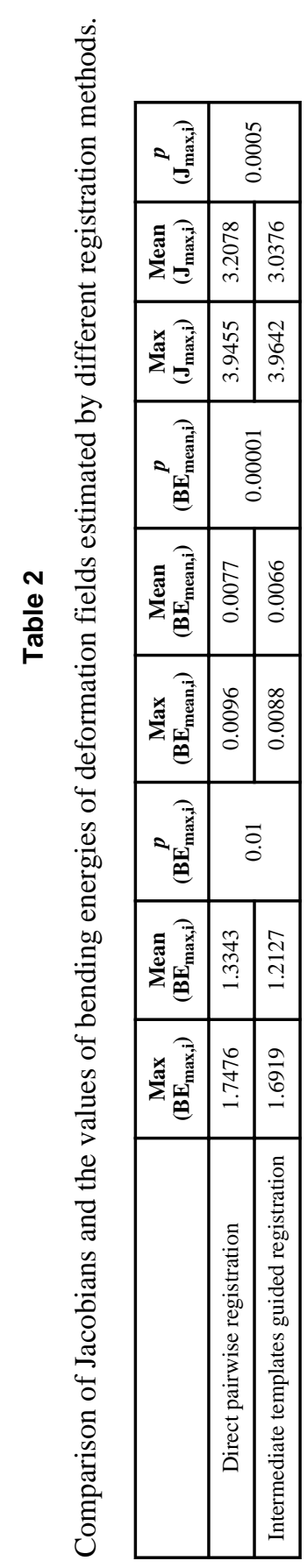




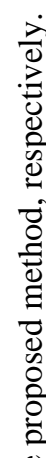

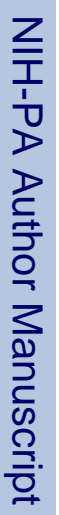

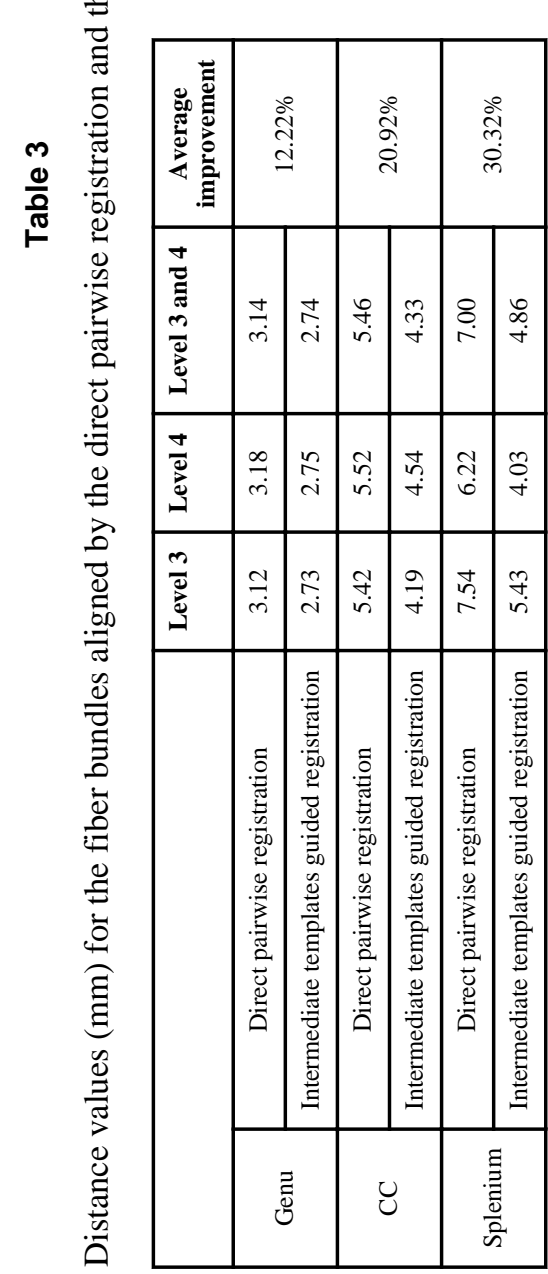

Neuroimage. Author manuscript; available in PMC 2012 January 15. 
Table 4

Fiber tracking on the mean diffusion tensor images.

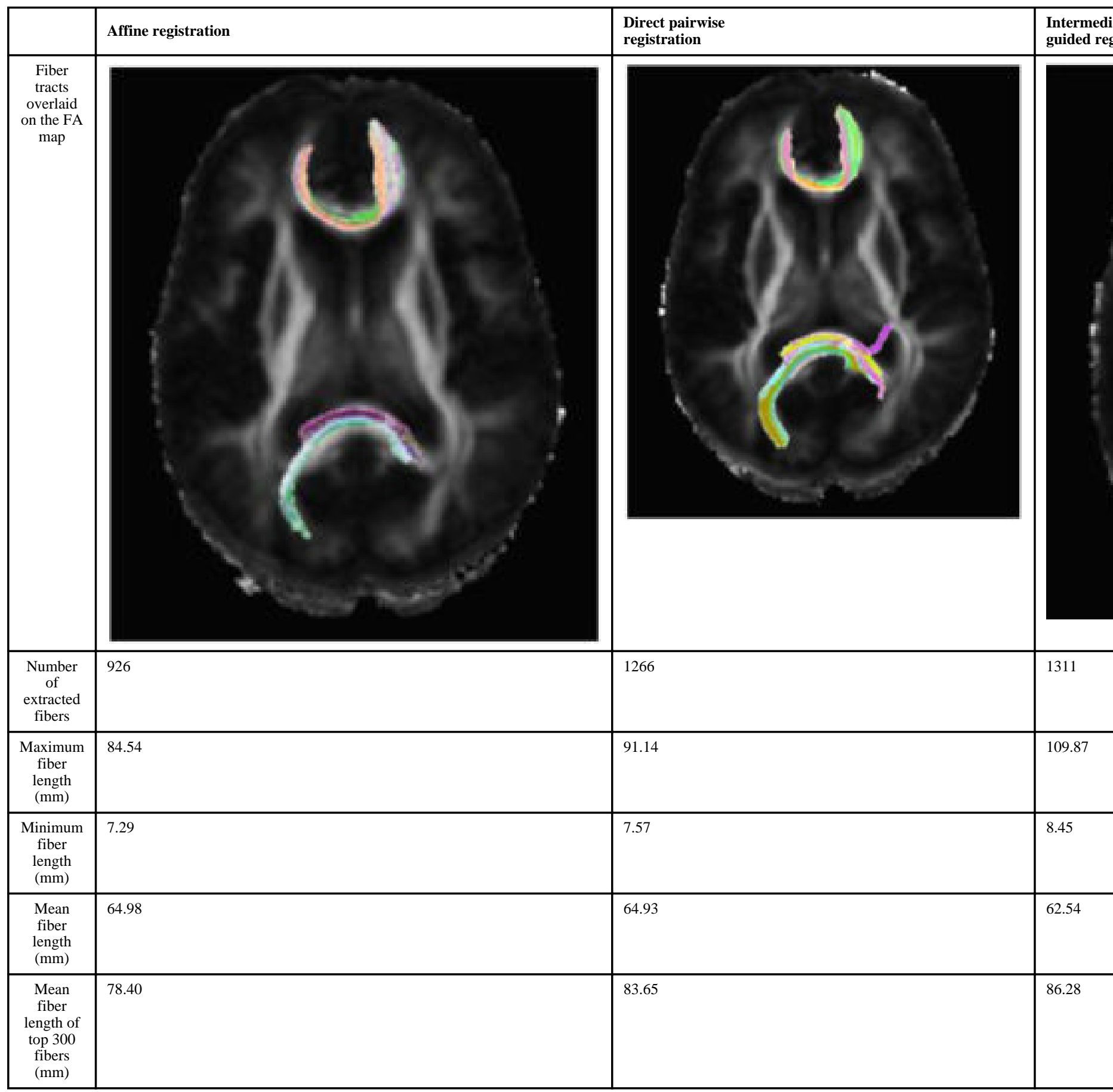

